\title{
The measurement results adjustment by the Least Square Method
}

\author{
Samoilenko Oleksandr ${ }^{1}$ (D), Kuzmenko Yurii ${ }^{2}$ \\ ${ }^{1,2} \mathrm{SE} \ll U k r m e t r t e s t s t a n d a r t »$, Ukraine \\ E-mail: asam@ukrcsm.kiev.ua
}

\begin{abstract}
The procedure for processing of the measurement results obtained from Comite International des Poids et Measures (CIPM) Key, Regional Metrology Organizations (RMO) or supplementary comparisons, from the proficiency testing by interlaboratory comparisons and the calibrations is proposed. It is named by authors as adjustment by the least square method (LSM). Additive and multiplicative parameters for each measurement standard of every particular laboratory will be the results of this adjustment, as well as the parameters for each artifact.

The parameters of the measurement standards are their additive and multiplicative degrees of equivalence from the comparison and the estimations of the systematic errors (biases) from calibrations. The parameters of the artifacts are the key comparisons reference value from the comparison and the assigned quantity values from the calibrations.

The adjustment is considered as a way to solving a problem of processing the great amount of homogeneous measurements with many measurement standards at a different comparison levels (CIPM, RMO or supplementary), including connected problems.

Four different cases of the adjustments are considered. The first one is a free case of adjustment. It was named so because of the fact that none of participants has any advantage except their uncertainties of measurements.

The second one is a fixed case of adjustment. Measuring results of RMO and supplementary comparisons are rigidly linked to additive and multiplicative parameters of measurement standards of particular laboratories participated in CIPM key comparisons.

The third one is a case of adjustment with dependent equations. This one is not so rigidly linked to the new comparisons results to previous or to some other comparisons as for fixed case. It means that the new results of comparisons are influenced by the known additive and multiplicative parameters and vice versa.

The fourth one is a free case of adjustment with additional summary equations. In that case certain checking equations are added to the system of equations. So, the sum of parameters multiplied by their weights of all measurement standards for particular laboratories participated in comparisons should be equal to zero.
\end{abstract}

Keywords: adjustment, least square method, key comparisons reference value, additive and multiplicative degrees of equivalence, parameters, measurement standard, uncertainty.

\section{Introduction}

It is widely accepted that the reliability of the results of Comite International des Poids et Measures (CIPM), Regional Metrology Organizations (RMO) key comparisons and supplementary comparison of measurement standards depends on the number of measurements that will be performed by the participants. The experience gives certain evidence that the more artifacts take part in the comparisons and the more measurements are carried out on participants' measurement standards in more points on the scale, the better results can be achieved. However, this leads to the problem of processing a rather large number of related measurement results. Such processing procedures are proposed here called by authors «the adjustment of the measurement results by the least square method (LSM)».

So, the aim of this publication is to present the mathematical apparatus for the adjustment 
of the comparisons measurement results by the Least Square Method. Developed mathematical apparatus can become a basis for the powerful software for adjustment of measurement results during international comparisons, the proficiency testing by interlaboratory comparisons and the calibrations.

The proposed mathematical apparatus are:

- multifunctional, i.e. suitable to adjustment of most homogeneous measurement results;

- mathematically strict, which gives the best estimations of the artifacts and measurement standards parameters and their uncertainties;

- simple and clear to users;

- suitable for creation of the software which will ignore the restrictions on the number of measurement results processed simultaneously;

- suitable for linking between CIPM key comparisons [1], RMO and supplementary comparisons for all complicated cases.

This publication focuses on the mathematical aspects of the processing of measurement results and does not concern the technical capability of the comparison participants and the expediency of using the same artifacts in several comparisons.

\section{Critical analysis of sources}

The processing method [2] for quantity value obtained for one artifact at one point by several measurement standards and estimation of the measurement uncertainty for this one quantity value according to [3] are particular cases of the proposed method of processing by LSM. The proposed measurement models $(2.48,[4])$ and their processing by LSM, are in general, similar to multivariate ones from $[5,6]$, but not in details.

The results of the analysis of sources had shown that the general method for determining the additive and multiplicative degrees of measurement standards equivalence and associated uncertainties was not clearly described.

The simplified equations in [7], [8], [9] are similar to the following below equations (1) and (2). It should be noted that for such a measurement model the rank of the coefficients matrix of the normal equations is less than the number of rows in it. Therefore, the matrix degenerates (the matrix determinant is equal to zero) and the system of equations has no solution. The method for overcoming this problem, given in [9], is not correct. It lies in the locking of the intercomparison loop. This means that the measurements should start and finish in the same laboratory. This approach does not appear to allow the expansion of the matrix rank to the full. A pseudo-inversion can be applied to solve the system of equations with incomplete rank [15].
It seems fair to point out that the sources do not describe the general method of linking the results of RMO key comparisons and supplementary comparisons to the CIPM key ones by fixing the reference quantity values of one or more artifacts and/or by fixing the degree of equivalence of one or more participants' reference standards. Methods of linking with CIPM key comparisons are given in [8], [10], [11] and [12], but without using LSM adjustment. It might be also pointed out that the methods given in [10], [11] and [12] are the particular cases of the proposed method, but they cannot solve the problem by taking into account all correlation connections.

It seems that nothing has been written about the use of a pseudo-inverse matrix for the solution of equations for the CIPM key comparisons when there are no fixed values. Here we'd like to mention the lack of discussion about the use of additional equations that can make it possible to expand the rank of the square coefficients matrix of normal equations to the full.

To describe the proposed procedure, some new terms have been established. Thus, the term measurement standards degree of equivalence [2] in the point of measurement has been supplemented into two important terms: additive and multiplicative parameters of measurement standards. Under them it is possible to understand the bias of zero of the measurement scale for measurement standard and the bias of measurement unit for measurement standard (1.9 [4]) respectively.

\section{Measurement model for determining artifacts param- eters and additive measurement standards parameters}

There are measurement standards that for certain reasons measure any quantity value with an additive and/or multiplicative systematic error (2.17, [4]). It means, for additive error, that a definite unknown constant is added to any measured value, as if there is a certain bias of the zero of the measurement scale for a given measurement standard with respect to its true but unknown position.

Accordingly, to any measured quantity value, a certain unknown quantity is added. This unknown quantity is proportional to the measured quantity value itself as if the scale for this measurement standard was slightly stretched or compressed. The estimation of this proportion is called hereinafter the multiplicative degree of the measurement standard equivalence and is obtained from the results of the comparisons.

Using the terminology and equations (3) from [2], and similar equations (2) from [8], equation (1) from [9], equation (2) from [13] and equation (47) from [14], one can theoretically state that:

$$
x_{i j}=y_{i}+d_{j}+b_{j} \cdot x_{i j}
$$


where

$x_{i j}$ is the quantity value measured by a measurement standard;

$y_{i}$ is the parameter of artifact, for example, key comparisons reference value that is assigned to an artifact;

$d_{j}, b_{j}$ is the additive and multiplicative parameters of the measurement standard, for example, the additive and multiplicative degrees of equivalence of the measurement standard;

$i=1 \ldots n$ is the number of artifacts and $j=1 . . . k$ is the number of measurement standards respectively.

In fact, before the adjustment, the values of $y_{i}$, $d_{j}$ and $b_{j}$ are the unknown and measured quantity values $x^{\prime}$ are weighed down by the random and systematic errors.

Taking into account the errors of the measurements by each measurement standard of each quantity value and the fact that $y_{i}, d_{j}$ and $b_{j}$ so far are unknown, the equation (1) we transform into an equation of corrections, omitting some intermediate considerations:

$$
v_{x_{i j}}=\delta y_{i}+\delta d_{j}+\delta b_{j} \cdot x_{i j}+l_{i j},
$$

where

$v_{x_{i j}}$ is the correction from the adjustment to the measured quantity value;

$\delta y_{i}, \delta d_{j}, \delta b_{j}$ are the corrections from adjustment to the initial quantity of the artifact parameter and to the initial quantity of the additive and multiplicative parameters of the measurement standard;

$l_{i j}=y_{0 i}+d_{0 j}+b_{0 j} \cdot x_{i j}-x_{i j}$ is the constant term of the equation of corrections;

$y_{0 i}, d_{0 j}, b_{0 j}$ are the initial quantity values of the artifact parameter and of the additive and multiplicative parameters of the measurement standard.

The adjustment allows you to evaluate the additive and multiplicative components of the measurement's systematic errors of each measurement standard. This procedure is weakening the influence of the measurement standards systematic errors to the evaluated parameters of artifact.

\section{Adjustment of the measurement results}

Adjustment is a processing procedure for all combinations of measurement results by LSM when performed the comparisons of the measurement standards, the proficiency testing by interlaboratory comparisons or the calibrations. All the considered below cases are solved following the main LSM condition:

$$
V_{x}^{T} \cdot W_{x} \cdot V_{x}=\min
$$

or in the particular case

$$
\sum_{i=1}^{n} \sum_{j=1}^{k} w_{i j} \cdot v_{x_{i j}}^{2}=\min
$$

where

$V_{x}$ is the vector of the corrections to the measured quantity values;

$W_{x}$ is the diagonal matrix of the weights of the measured quantity values.

Measurement standards of different accuracy levels can be involved in comparisons. Level of accuracy must be taken into account by the corresponding weight coefficients (weights) $w_{i j}$ of the measurement results prior their adjustment:

$$
w_{i j}=\frac{\sigma_{0}^{2}}{u\left(x_{i j}\right)^{2}},
$$

where

$u\left(x_{i j}\right)=\sigma_{0}+\delta_{0} \cdot x_{i j}$ is standard uncertainty of the measured quantity values;

$\sigma_{0}$ is standard absolute uncertainties of some measurements, for which the weight is set to unity: $w_{0}=\sigma_{0}^{2} / u\left(x_{i j}\right)^{2}=1$;

$\delta_{0}$ is standard relative uncertainties of some measurements, for which the weight is set to unity: $w_{\delta_{0}}=\sigma_{0}^{2} / \delta_{0}^{2} \cdot x_{i j}^{2}=1$.

The weights are the dimensionless proportionality coefficients that increase the effect of more accurate measurements on the final result and reduce the effect of less accurate ones.

Uncertainty of the measurement results in formula (3) may be evaluated by the other way.

Four cases of the adjustment are considered:

A. Adjustment of the freedom case;

B. Adjustment of the fixed case;

C. Adjustment of the dependent equations case;

D. Adjustment of the freedom case with the additional summary equations.

The reason for the freedom case name is that no participants' measurement result has any advantage besides their uncertainties of measurements. This case is useful for the adjustment of the CIPM key comparisons measurements when any parameter, calculated from the equations solution, is not fixed. Only uncertainty of measurement results through their weights have influence on results of adjustment.

On the contrary to the freedom case, the fixed case is named so due to the fact that one or few of the parameters of artifacts and/or the parameters 
of the measurement standards must not be changed during the adjustment. These parameters are well known, for example, from CIPM key comparisons before the data adjustment of the RMO or supplementary comparisons.

The dependent equations case is named so because it combines both the freedom and the fixed cases. One or few, or all of the reference quantity values and/or the participants' parameters are known and have uncertainties from previous or other comparisons. Each of these dependent parameters gives the additional equations to the system of equations. Old and new parameters, when they are adjusted, influence each other proportionally to their weights. It is important to note that fixed and dependent cases can be mixed in all combinations.

The freedom case with additional summary equations is more important for practice. It is named so, due to the fact that the system of equations is complemented with one or two summary equations similar to (13). In such equations, the sums of the additive parameters and, separately, multiplicative parameters after adjustment must be equal to zero. That is why such equations are called here «summary equations». Such an extended system of equations allows averaging the zero of scale and measurement unit from all the adjusted measurement results.

\section{Adjustment of the freedom case}

This approach can be used when parameters shall be defined from the adjustment. Herewith, the adjustment is fulfilled with conditions:

$$
\sum_{j=1}^{k} d_{j}^{2}=\min ; \quad \sum_{j=1}^{k} b_{j}{ }^{2}=\min .
$$

For the purpose of the adjustment of the measurement results by means of LSM, the equation (2) for the freedom case in the general matrix form is as follows:

$$
V_{x}=\left[\begin{array}{lll}
A_{y} & A_{d} & A_{b}
\end{array}\right]\left[\begin{array}{l}
\delta y \\
\delta d \\
\delta b
\end{array}\right]+l,
$$

where

$A_{y}, A_{d}, A_{b}$ are the matrixes of the coefficients of the corrections equations at the artifact parameters, the additive and multiplicative parameters of measurement standards;

$\delta y, \delta d, \delta b$ are the column vectors of the corrections to the initial values regarding the artifact parameters, the additive and multiplicative parameters of the measurement standards;

$l$ is the column vector of the constant terms of the corrections equations.
The normal equations are as follows:

$$
\left[\begin{array}{ccc}
N_{y} & N_{12} & N_{13} \\
N_{21} & N_{d} & N_{23} \\
N_{31} & N_{32} & N_{b}
\end{array}\right] \cdot\left[\begin{array}{c}
\delta y \\
\delta d \\
\delta b
\end{array}\right]+\left[\begin{array}{c}
L_{y} \\
L_{d} \\
L_{b}
\end{array}\right]=0
$$

where

$$
\begin{gathered}
{\left[\begin{array}{ccc}
N_{y} & N_{12} & N_{13} \\
N_{21} & N_{d} & N_{23} \\
N_{31} & N_{32} & N_{b}
\end{array}\right]=\left[\begin{array}{lll}
A_{y}^{T} W_{x} A_{y} & A_{y}^{T} W_{x} A_{d} & A_{y}^{T} W_{x} A_{b} \\
A_{d}^{T} W_{x} A_{y} & A_{d}^{T} W_{x} A_{d} & A_{d}^{T} W_{x} A_{b} \\
A_{b}^{T} W_{x} A_{y} & A_{b}^{T} W_{x} A_{d} & A_{b}^{T} W_{x} A_{b}
\end{array}\right] ;} \\
{\left[\begin{array}{l}
L_{y} \\
L_{d} \\
L_{b}
\end{array}\right]=\left[\begin{array}{l}
A_{y}^{T} W_{x} l \\
A_{d}^{T} W_{x} l \\
A_{b}^{T} W_{x} l
\end{array}\right] .}
\end{gathered}
$$

For the freedom case the matrixes of coefficients of the normal equations (5) under the inversion are degenerated, because the rank of the matrix of normal equations is incomplete and the determinant of the system of normal equations is equal to zero. To overcome degeneration of the normal equations matrix the matrix pseudo-inverse method described in [15] is used to find the solution:

$$
\begin{aligned}
{\left[\begin{array}{l}
\delta y \\
\delta d \\
\delta b
\end{array}\right] } & =-\left[\begin{array}{lll}
N_{y} & N_{12} & N_{13} \\
N_{21} & N_{d} & N_{23} \\
N_{31} & N_{32} & N_{b}
\end{array}\right]^{+} \cdot\left[\begin{array}{c}
L_{y} \\
L_{d} \\
L_{b}
\end{array}\right]= \\
& =-\left[\begin{array}{lll}
Q_{y} & Q_{12} & Q_{13} \\
Q_{21} & Q_{d} & Q_{23} \\
Q_{31} & Q_{32} & Q_{b}
\end{array}\right] \cdot\left[\begin{array}{c}
L_{y} \\
L_{d} \\
L_{b}
\end{array}\right],
\end{aligned}
$$

where

$$
\left[\begin{array}{lll}
Q_{y} & Q_{12} & Q_{13} \\
Q_{21} & Q_{d} & Q_{23} \\
Q_{31} & Q_{32} & Q_{b}
\end{array}\right]=\left(\left[\begin{array}{ccc}
N_{y} & N_{12} & N_{13} \\
N_{21} & N_{d} & N_{23} \\
N_{31} & N_{32} & N_{b}
\end{array}\right]\right)^{+} \text {is the matrix }
$$
pseudo-inversed to the normal equations matrix.

\section{Adjustment of the fixed case}

For the adjustment of the RMO key comparisons and supplementary comparison results, the results of the interlaboratory comparisons or the calibrations of one or more parameters for the artifacts and/or the additive and/or multiplicative parameters of measurement standards obtained from CIPM key comparisons are fixed:

$$
y_{i}^{c}=\text { const } \text { and/or } d_{j}^{c}=\text { const } \text { and/or } b_{j}^{c}=\text { const } .
$$

Taking into account the equations (7), the equations of corrections (2) related to $y_{i}^{c}$ or $d_{j}^{c}$ or $b_{j}^{c}$ accordingly for the fixed cases are as follows: 


$$
\begin{gathered}
v_{x_{i j}}=\delta d_{j}+\delta b_{j} \cdot x_{i j}+l_{i j} ; l_{i j}=y_{i}^{c}+d_{0 j}+b_{0 j} \cdot x_{i j}-x_{i j} ; \\
v_{x_{i j}}=\delta y_{i}+\delta b_{j} \cdot x_{i j}+l_{i j} ; l_{i j}=y_{0 i}+d_{j}^{c}+b_{0 j} \cdot x_{i j}-x_{i j} ; \\
v_{x_{i j}}=\delta y_{i}+\delta d_{j}+l_{i j} ; l_{i j}=y_{0 i}+d_{0 j}+b_{j}^{c} \cdot x_{i j}-x_{i j} .
\end{gathered}
$$

The equations of corrections (2) related to $y_{i}^{c}$ and $d_{j}^{c}$, or $d_{j}^{c}$ and $b_{j}^{c}$, or $y_{i}^{c}$ and $b_{j}^{c}$ are as follows:

$$
\begin{gathered}
v_{x_{i j}}=\delta b_{j} \cdot x_{i j}+l_{i j} ; l_{i j}=y_{i}^{c}+d_{j}^{c}+b_{0 j} \cdot x_{i j}-x_{i j} ; \\
v_{x_{i j}}=\delta y_{i}+l_{i j} ; l_{i j}=y_{0 i}+d_{j}^{c}+b_{j}^{c} \cdot x_{i j}-x_{i j} ; \\
v_{x_{i j}}=\delta d_{j}+l_{i j} ; l_{i j}=y_{i}^{c}+d_{0 j}+b_{j}^{c} \cdot x_{i j}-x_{i j} .
\end{gathered}
$$

The equation of corrections (2) related to $y_{i}^{c}, d_{j}^{c}$ and $b_{j}^{c}$ simultaneously are as follows:

$$
v_{x_{i j}}=l_{i j} ; l_{i j}=y_{i}^{c}+d_{j}^{c}+b_{j}^{c} \cdot x_{i j}-x_{i j} \text {. }
$$

Equations (8) suppose that one or more values are considered unchanged (uncorrected). Therefore, this case is called «fixed case». This means that one or more participants have an advantage.

Approximated lines for these equations will be passing through the point $y_{i}^{c}=$ const and/or the lines will cross the axis of $x$ in the point $d_{j}^{c}=$ const and/or the tilt of lines will be equal $b_{j}^{c}=$ const.

For the purposes of RMO key comparisons and supplementary comparisons, when one or more artifacts and/or measurement standards of participants were also involved in the CIPM key comparisons, the linking problem to the related CIPM key comparisons is solved automatically considering all correlation connections. The application of the fixed case leads to the fact that all artifacts' parameters and measurement standards' additive and multiplicative parameters will be rigidly linked to values from the CIPM key comparisons. After adjustment the results of the interlaboratory comparisons or the calibrations will be rigidly linked to the CIPM key comparisons too.

\section{Adjustment of the dependent equations case}

To our opinion, adjustment of the dependent equations case of the CIPM key comparisons, RMO key comparisons and supplementary comparisons results lays between two previous cases. The idea is to process the measurements taking into account the results of previous (or other) comparisons, but without rigid binding/fixing, as under the conditions of the fixed cases. For this reason, the following dependent equations are added to the system of equations (1):

$$
\dot{y}_{i}=y_{i} \text { and/or } \dot{d}_{j}=d_{j} \text { and/or } \dot{b}_{j}=b_{j} \text {, }
$$

where

$\dot{y}_{i}, \quad \dot{d}_{j}, \dot{b}_{j}$ are the quantities of the artifacts parameters and of the parameters of the measurement standards obtained from previous comparisons;

$y_{i}, d_{j}, b_{j}$ are the unknown parameters of artifacts and the unknown additive and multiplicative parameters of the measurement standards, the same that in the equation (1).

For this case the results of the adjustment depends on the weights (12) assigned to $\dot{y}_{i}, \dot{d}_{j}$ and $\dot{b}_{j}$ from the CIPM key comparisons and/or RMO key comparisons in any combination. If the weights are too small, the solution will be close to the freedom case, if the weights are too big, the solution will be close to the fixed case.

On the basis of (9), the additional dependent correction equations are added to the system of equations of corrections (2) as follows:

$$
\begin{gathered}
v_{\dot{y}_{i}}=\delta y_{i}+l_{\dot{y}_{i}} ; l_{\dot{y}_{i}}=y_{0 i}-\dot{y}_{i} ; \\
v_{\dot{d}_{j}}=\delta d_{j}+l_{\dot{d}_{j}} ; l_{\dot{d}_{j}}=d_{0 j}-\dot{d}_{j} ; \\
v_{\dot{b}_{j}}=\delta b_{j}+l_{\dot{b}_{j}} ; l_{\dot{b}_{j}}=b_{0 j}-\dot{b}_{j},
\end{gathered}
$$

where

$v_{\dot{y}_{i}}, v_{\dot{d}_{j}}, v_{\dot{b}_{j}}$ are the corrections from adjustment to the quantity of the artifact parameters and to the parameters of the measurement standard, which was obtained from previous comparisons.

The adding of at least one of the equations (10) complements the matrix of normal equations to full rank and makes the solution of the system of normal equations not degenerate.

For the adjustment under the dependent equations case, the equation (2) with the additional dependent correction equations from previous comparisons (10) in the general matrix form is as follows:

$$
\left[\begin{array}{cccc}
V_{x} & 0 & 0 & 0 \\
0 & V_{\dot{y}} & 0 & 0 \\
0 & 0 & V_{\dot{d}} & 0 \\
0 & 0 & 0 & V_{\dot{b}}
\end{array}\right]=\left[\begin{array}{ccc}
A_{y} & A_{d} & A_{b} \\
B_{y} & 0 & 0 \\
0 & B_{d} & 0 \\
0 & 0 & B_{b}
\end{array}\right] \cdot\left[\begin{array}{c}
\delta y \\
\delta d \\
\delta b
\end{array}\right]+\left[\begin{array}{c}
l \\
l_{\dot{y}} \\
l_{\dot{d}} \\
l_{\dot{b}}
\end{array}\right],
$$

where

$B_{y}=E, B_{d}=E, B_{b}=E$ are the matrixes of the coefficients of the additional dependent correction equations;

$l_{\dot{y}}, l_{\dot{d}}, l_{\dot{b}}$ are the columns vectors of the constant terms of the equations (10);

$V_{\dot{y}}, V_{\dot{d}}, V_{\dot{b}}$ are the diagonal matrixes of corrections to the quantity of the artifacts parameters 
and the parameters of the measurement standard obtained from previous comparisons. trixes:

The normal equations (4) have the following ma-

$$
\begin{gathered}
{\left[\begin{array}{ccc}
N_{y} & N_{12} & N_{13} \\
N_{21} & N_{d} & N_{23} \\
N_{31} & N_{32} & N_{b}
\end{array}\right]=} \\
=\left[\begin{array}{ccc}
A_{y}^{T} W_{x} A_{y}+B_{y}^{T} W_{\dot{y}} B_{y} & A_{y}^{T} W_{x} A_{d} & A_{y}^{T} W_{x} A_{b} \\
A_{d}^{T} W_{x} A_{y} & A_{d}^{T} W_{x} A_{d}+B_{d}^{T} W_{\dot{d}} B_{d} & A_{d}^{T} W_{x} A_{b} \\
A_{b}^{T} W_{x} A_{y} & A_{b}^{T} W_{x} A_{d} & A_{b}^{T} W_{x} A_{b}+B_{b}^{T} W_{\dot{b}} B_{b}
\end{array}\right] ; \\
{\left[\begin{array}{c}
L_{y} \\
L_{d} \\
L_{b}
\end{array}\right]=\left[\begin{array}{c}
A_{y}^{T} W_{x} l+B_{y}^{T} W_{\dot{y}} l_{\dot{y}} \\
A_{d}^{T} W_{x} l+B_{d}^{T} W_{\dot{d}} l_{\dot{d}} \\
A_{b}^{T} W_{x} l+B_{b}^{T} W_{\dot{b}} l_{\dot{b}}
\end{array}\right],}
\end{gathered}
$$

where

$W_{\dot{y}}, W_{\dot{d}}, W_{\dot{b}}$ are the weights matrix of the artifacts parameters and the weights matrixes of the additive and multiplicative parameters of the measurement standards, obtained from previous or other comparisons are calculated according to:

$$
w_{\dot{y}_{i}}=\frac{\sigma_{0}^{2}}{u\left(\dot{y}_{i}\right)^{2}} \text { or } w_{\dot{d}_{j}}=\frac{\sigma_{0}^{2}}{u\left(\dot{d}_{j}\right)^{2}} \text { or } w_{\dot{b}_{j}}=\frac{\delta_{0}^{2}}{u\left(\dot{b}_{j}\right)^{2}},
$$

where

$u\left(\dot{y}_{i}\right), u\left(\dot{d}_{j}\right)$ and $u\left(\dot{b}_{j}\right)$ are the standard uncertainties of the artifact parameters and the additive and multiplicative parameters of the measurement standards, obtained from previous or another comparisons.

\section{Adjustment of the freedom case with additional summary equations}

The idea of this case is to process the measurement results taking into account a priori information about the correlation connections between the comparison reference value and the parameters. For unequal case of the parameters to fulfill these conditions, the additional summary equations are required:

$$
\sum_{j=1}^{k} \bar{w}\left(d_{j}\right) \cdot d_{j}=0 \text { and/or } \sum_{j=1}^{k} \bar{w}\left(b_{j}\right) \cdot b_{j}=0 .
$$

The conditions in (13) are not fulfilled for the cases of adjustment described in sections 5, 6 and 7 . Considering (13) for the freedom case with additional summary equations, summary equations of corrections are added to the system of equations of corrections (2) as follows:

$$
\begin{gathered}
v_{\Sigma d}=\sum_{j=1}^{k} \bar{w}\left(d_{j}\right) \cdot \delta d_{j}+l_{\Sigma d} ; l_{\Sigma d}=\sum_{j=1}^{k} \bar{w}\left(d_{j}\right) \cdot d_{0 j} . \\
v_{\Sigma b}=\sum_{j=1}^{k} \bar{w}\left(b_{j}\right) \cdot \delta b_{j}+l_{\Sigma b} ; l_{\Sigma b}=\sum_{j=1}^{k} \bar{w}\left(b_{j}\right) \cdot b_{0 j} .
\end{gathered}
$$

In the equation (13), the weights for the parameters $\bar{w}\left(d_{j}\right)$ and $\bar{w}\left(b_{j}\right)$ are the coefficients of the correction equations. The weights $\bar{w}\left(d_{j}\right)$ and $\bar{w}\left(b_{j}\right)$ are obtained analogically to formulas (12).

For the adjustment of the freedom case with additional summary equations the results of measurements under LSM, the equation (11) with correction equations (14) in the general matrix form is as follows:

$$
\left[\begin{array}{ccc}
V_{x} & 0 & 0 \\
0 & v_{\Sigma d} & 0 \\
0 & 0 & v_{\Sigma b}
\end{array}\right]=\left[\begin{array}{ccc}
A_{y} & A_{d} & A_{b} \\
0 & \bar{B}_{d} & 0 \\
0 & 0 & \bar{B}_{b}
\end{array}\right] \cdot\left[\begin{array}{c}
\delta y \\
\delta d \\
\delta b
\end{array}\right]+\left[\begin{array}{c}
l \\
l_{\Sigma d} \\
l_{\Sigma b}
\end{array}\right],
$$

where

$\bar{B}_{d}, l_{\Sigma d}, v_{\Sigma d}$ is the vector of the coefficients, constant term and correction of the additional summary correction equations for the additive parameters;

$\bar{B}_{b}, l_{\Sigma b}, v_{\Sigma b}$ is the vector of the coefficients, constant term and correction of the additional summary correction equations for the multiplicative parameters.

Vectors of the coefficients of the additional summary correction equations to the equations system (11) are as follows:

$$
\begin{aligned}
\bar{B}_{d} & =\left[\begin{array}{lllll}
\bar{w}\left(d_{1}\right) & \ldots & \bar{w}\left(d_{j}\right) & \ldots & \bar{w}\left(d_{k}\right)
\end{array}\right] ; \\
\bar{B}_{b} & =\left[\begin{array}{lllll}
\bar{w}\left(b_{1}\right) & \ldots & \bar{w}\left(b_{j}\right) & \ldots & \bar{w}\left(b_{k}\right)
\end{array}\right] .
\end{aligned}
$$

The normal equations (4) have the following matrixes:

$$
\left[\begin{array}{lll}
N_{y} & N_{12} & N_{13} \\
N_{21} & N_{d} & N_{23} \\
N_{31} & N_{32} & N_{b}
\end{array}\right]=
$$

$$
=\left[\begin{array}{ccc}
A_{y}^{T} W_{x} A_{y} & A_{y}^{T} W_{x} A_{d} & A_{y}^{T} W_{x} A_{b} \\
A_{d}^{T} W_{x} A_{y} & A_{d}^{T} W_{x} A_{d}+\bar{B}_{d}^{T} \bar{B}_{d} & A_{d}^{T} W_{x} A_{b} \\
A_{b}^{T} W_{x} A_{y} & A_{b}^{T} W_{x} A_{d} & A_{b}^{T} W_{x} A_{b}+\bar{B}_{b}^{T} \bar{B}_{b}
\end{array}\right] ;
$$

$$
\left[\begin{array}{c}
L_{y} \\
L_{d} \\
L_{b}
\end{array}\right]=\left[\begin{array}{c}
A_{y}^{T} W_{x} l \\
A_{d}^{T} W_{x} l+\bar{B}_{d}^{T} l_{\Sigma d} \\
A_{b}^{T} W_{x} l+\bar{B}_{b}^{T} l_{\Sigma b}
\end{array}\right] .
$$


The matrixes $N_{d}$ in blocks of the matrix of the normal equations (15) have the form:

$$
N_{d}=\left[\begin{array}{ccccc}
\sum_{i=1}^{n} w_{i 1}+\bar{w}^{2}\left(d_{1}\right) & \ldots & \bar{w}\left(d_{1}\right) \bar{w}\left(d_{j}\right) & \ldots & \bar{w}\left(d_{1}\right) \bar{w}\left(d_{k}\right) \\
\ldots & \ldots & \ldots & \ldots & \ldots \\
\bar{w}\left(d_{j}\right) \bar{w}\left(d_{1}\right) & \ldots & \sum_{i=1}^{n} w_{i j}+\bar{w}^{2}\left(d_{j}\right) & \ldots & \bar{w}\left(d_{j}\right) \bar{w}\left(d_{k}\right) \\
\ldots & \ldots & \ldots & \ldots & \ldots \\
\bar{w}\left(d_{k}\right) \bar{w}\left(d_{1}\right) & \ldots & \bar{w}\left(d_{k}\right) \bar{w}\left(d_{j}\right) & \ldots & \sum_{i=1}^{n} w_{i k}+\bar{w}^{2}\left(d_{k}\right)
\end{array}\right]
$$

The matrixes $N_{b}$ in blocks of the matrix of the normal equations (15) have the form:

$$
N_{b}=\left[\begin{array}{ccccc}
\sum_{i=1}^{n} w_{i 1}+\bar{w}^{2}\left(b_{1}\right) & \ldots & \bar{w}\left(b_{1}\right) \bar{w}\left(b_{j}\right) & \ldots & \bar{w}\left(b_{1}\right) \bar{w}\left(b_{k}\right) \\
\ldots & \ldots & \ldots & \ldots & \ldots \\
\bar{w}\left(b_{j}\right) \bar{w}\left(b_{1}\right) & \ldots & \sum_{i=1}^{n} w_{i j}+\bar{w}^{2}\left(b_{j}\right) & \ldots & \bar{w}\left(b_{j}\right) \bar{w}\left(b_{k}\right) \\
\ldots & \ldots & \ldots & \ldots & \ldots \\
\bar{w}\left(b_{k}\right) \bar{w}\left(b_{1}\right) & \ldots & \bar{w}\left(b_{k}\right) \bar{w}\left(b_{j}\right) & \ldots & \sum_{i=1}^{n} w_{i k}+\bar{w}^{2}\left(b_{k}\right)
\end{array}\right]
$$

In this partial equal case, when $u\left(d_{1}\right)=u\left(d_{2}\right)=\ldots=$ $=u\left(d_{k}\right)=u_{0}(d)$ and $u\left(b_{1}\right)=u\left(b_{2}\right)=\ldots=u\left(b_{k}\right)=u_{0}(b)$, the matrixes (16) and (17) are as follows:

$$
\begin{aligned}
& \bar{B}_{d}^{T} \bar{B}_{d}=\frac{\sigma_{0}^{2}}{u_{0}^{2}(d)} \cdot\left[\begin{array}{cccc}
1 & 1 & \ldots & 1 \\
1 & 1 & \ldots & 1 \\
\ldots & \ldots & \ldots & \ldots \\
1 & 1 & \ldots & 1
\end{array}\right] ; \\
& \bar{B}_{b}^{T} \bar{B}_{b}=\frac{\delta_{0}^{2}}{u_{0}^{2}(b)} \cdot\left[\begin{array}{cccc}
1 & 1 & \ldots & 1 \\
1 & 1 & \ldots & 1 \\
\ldots & \ldots & \ldots & \ldots \\
1 & 1 & \ldots & 1
\end{array}\right] .
\end{aligned}
$$

The adding of the equations (14) complements the matrix of normal equations to full rank and makes the solution of the system of normal equations not degenerate.

The adding of equation (13) to the equations system (1) is the only way to average the zero of scale and the measurement unit on the scale that is realized by the participants' specific measurement standards. All additive and multiplicative parameters for participants' specific measurement standards are obtained in regard to this average zero and average measurement unit.

\section{Uncertainty estimation from adjustment}

Uncertainty estimation for all cases of adjustment mentioned above is described in this section.
The standard deviation of some measurements, for which the weight is set to unity is calculated as follows:

$$
S=\sqrt{\frac{\sum_{i=1}^{n} \sum_{j=1}^{k} w_{i j} \cdot v_{x_{i j}}^{2}+\sum_{i=1}^{n} w_{\dot{y}_{i}} \cdot v_{\dot{y}_{i}}^{2}+\sum_{j=1}^{k} w_{d_{j}} \cdot v_{d_{j}}^{2}+\sum_{j=1}^{k} w_{b_{j}} \cdot v_{b_{j}}^{2}}{r}},
$$

where

$r$ is the degree of freedom number.

The type A uncertainties for the quantity of the artifact parameters, and of the measurement standards additive and multiplicative parameters are calculated by the formulas:

$$
u_{A}\left(y_{i}\right)=S \cdot \sqrt{Q_{y_{i i}}} ; u_{A}\left(d_{j}\right)=S \cdot \sqrt{Q_{d_{j j}}} ; \quad u_{A}\left(b_{j}\right)=S \cdot \sqrt{Q_{b_{j j}}} .
$$

Additive and multiplicative degrees of equivalence of each measurement standard are obtained from the adjustment of both the relative averaged zero of the scale and the averaged measurement unit. The zero of the scale type A uncertainties and the measurement unit that is realized in the certain measurement range by all measurement standards involved in the comparison are evaluated using the weighting functions as follows:

$$
F_{\bar{d}}=\frac{\sum_{j=1}^{k} d_{j}}{k} ; F_{\bar{b}}=\frac{\sum_{j=1}^{k} b_{j}}{k} .
$$

Inverse weight of these weighting functions is calculated as follows:

$$
Q_{\bar{d}}=f_{\bar{d}} \cdot Q \cdot f_{\bar{d}}^{T} ; Q_{\bar{b}}=f_{\bar{b}} \cdot Q \cdot f_{\bar{b}}^{T}
$$

where

$f_{\bar{d}}=[1 / k \ldots 1 / k \ldots 1 / k]$ is the vector of the additive degrees of equivalence obtained from the partial derivatives of the first equation of (18);

$f_{\bar{b}}=[1 / k \ldots 1 / k \ldots 1 / k]$ is the vector of the multiplicative degrees of equivalence obtained from the partial derivatives of the second equation of (18);

$Q$ is the matrix calculated on the analogy to (6) inversed to the matrix of normal equations $\mathrm{N}$.

The zero of the scale type A uncertainties and the measurement unit shall be calculated as follows:

$$
u_{A}(\bar{d})=S \cdot \sqrt{Q_{\bar{d}}} ; u_{A}(\bar{b})=S \cdot \sqrt{Q_{\bar{b}}} \text {. }
$$

\section{Conclusions}

1. The adjustment by LSM is a mathematically strict and multi-purpose procedure of the measure- 
ment results processing, which is sufficiently simple to develop an appropriate software, which will help to process all the complicated combinations of a large number of the measurement results to obtain the parameters of artifacts and measurement standards.

2. The adjustment by LSM allows evaluating the generalized characteristics of the measurement standards, named in this article the additive and multiplicative parameters. These characteristics can be used to calculate corrections to the measurement results.

3. The additive parameter of measurement standard is the absolute bias of their entire measurements regarding to the zero of the measurement scale that is averaged after the adjustment of all measurements from the CIPM key comparison.

4. The multiplicative parameter of measurement standard is the relative bias of their entire measurements regarding to the unit of the measurement scale that is averaged after the adjustment of all measurements from the CIPM key comparison.

5. The freedom case of adjustment to obtain the artifacts parameters and measurement standards pa- rameters when none of them is fixed (not accepted as unchanged) has been proposed.

6. The fixed case of adjustment for processing RMO key comparisons and supplementary comparisons, under which the parameters for artifacts that participated in CIPM key comparisons are fixed (accepted as unchanged) and/or the parameters of measurement standards that participated in the CIPM key comparisons are fixed, has been proposed.

7. The dependent equations case of adjustment to obtain the artifact parameters and measurement standards parameters taking into account the parameters obtained from the previous CIPM or other comparisons have been proposed.

8. The freedom case with additional summary equations for averaging the zero of scale and the measurement unit of the scale that is realized by the participants' specific measurement standards has been proposed. All additive and multiplicative parameters for participants' specific measurement standards are obtained regarding to this average zero and average measurement unit.

\section{References}

1. MRA: Mutual recognition of national measurement standards and calibration and measurement certificate issued by national metrology institutes. International Committee for Weights and Measures. 14 October 1999, p. 45. www.bipm.org/pdf/mra.pdf

2. Cox M. G. 2002 The evaluation of key comparison data. Metrologia. 39, pp. 589-595. https://iopscience.iop.org/article/10.1088/0026-1394/ $\underline{39 / 6 / 10}$

3. JCGM 100:2008, Evaluation of Measurement Data-Guide to the Expression of Uncertainty in Measurement. Joint Committee for Guides in Metrology (JCGM).

4. JCGM 200:2012, International Vocabulary of Metrology Basic and General Concepts and Associated Terms (VIM). Joint Committee for Guides in Metrology (JCGM).

5. JCGM 102:2011 Evaluation of measurement data - Supplement 2 to the «Guide to the expression of uncertainty in measurement» Extension to any number of output quantities. Joint Committee for Guides in Metrology (JCGM).

6. Nielsen L. 2003 Identification and handling of discrepant measurements in key comparisons. Measurement Techniques. 46(5), pp. 513-522.

https://doi.org/10.1023/A:1025373701977.

7. Sutton C. M. 2004 Analysis and linking of international measurement comparisons Metrologia. 41, pp. 272-277.

http://iopscience.iop.org/0026-1394/41/4/008

8. Elster C., Chunovkina A. G., Woger W. 2010 Linking of a RMO key comparison to a related CIPM key comparison using the parameters of the linking laboratories. Metrologia. 47. http://iopscience.iop.org/0026-1394/10/010096
9. Koo A., Clare J. F. 2012 On the equivalence of generalized least-squares approaches to the evaluation of measurement comparisons. Metrologia. 49, pp. 340-348.

http://iopscience.iop.org/article/10.1088/0026-1394/ $\underline{49 / 3 / 340}$

10. White D. R. 2004 On the analysis of measurement comparisons. Metrologia. 41, pp. 122-131. iopscience.iop.org/0026-1394/41/3/003

11. Velychko O. M. 2009 Estimation of data of international comparisons of national standards: basic requirements and procedures. [Оброблення даних міжнародних звірень національних еталонів: основні вимоги і процедури] Ukrainian Metrological Journal. 3, pp. 57-63 [In Ukrainian].

12. COOMET R/GM/14:2006 2008 Guide on Estimation of the COOMET Key Comparison Data [Руководство по оцениванию результатов ключевых сличений КОOMET] (St. Petersburg: COOMET) p. 13. [In Russian].

13. Elster C., Toman B. 2013 Analysis of key comparison data: critical assessment of elements of current practice with suggested improvement. Metrologia. 50, p. 549

http://iopscience.iop.org/0026-394/13/050549

14. Kuzmenko Yu., Samoylenko O. 2018 Processing by least square method of the measurement results for key, regional and supplementary comparison of the measurement standards. [Опрацювання методом найменших квадратів результатів вимірювань за ключових, регіональних та додаткових звірень еталонів] Metrology and Devices. 70, pp. 3-13 [In Ukrainian].

15. Lawson C., Henson R. 1986 Solving Least Squares Problems. Science. Head Editor phys.-mat. lit., p. 232 [Trans. from English]. 